\title{
Distinctive Rorschach profiles of young adults with schizophrenia and autism spectrum disorder
}

\author{
This article was published in the following Dove Press journal: \\ Neuropsychiatric Disease and Treatment \\ 21 September 2016 \\ Number of times this article has been viewed
}

\author{
Naoko Kishimoto' \\ Kazuhiko Yamamuro' \\ Junzo lida ${ }^{2}$ \\ Toyosaku Ota' \\ Shohei Tanaka' \\ Masanori Kyo ${ }^{3}$ \\ Sohei Kimoto' \\ Toshifumi Kishimoto' \\ 'Department of Psychiatry, ${ }^{2}$ Faculty \\ of Nursing, Nara Medical University \\ School of Medicine, ${ }^{3}$ Department \\ of Psychiatry, Kyo Mental Clinic, \\ Nara, Japan
}

Correspondence: Sohei Kimoto Department of Psychiatry, Nara Medical University School of Medicine, 840 Shijyou-cho, Kashihara, Nara 634-8521, Japan

Tel +8I 74422305 I

Fax +8I 744223854

Email sohei@naramed-u.ac.jp
Objective: The differential diagnosis of schizophrenia (SZ) versus autism spectrum disorder (ASD) can be clinically challenging because accumulating evidence suggests both clinical and biological overlaps between them. The aim of this study was to compare Rorschach profiles between young adults with $\mathrm{SZ}$ and those with ASD.

Methods: We evaluated quantitative tendencies on the Rorschach test among 20 patients diagnosed with SZ and 20 diagnosed with ASD. Both groups were matched for age, sex, and intelligence quotient.

Results: We found significant differences in six response variables on the Rorschach comprehensive system. Those with SZ had significantly higher scores on D score, adjusted D score (Adj D), developmental quality code reflecting ordinary response (DQo), and form quality minus (FQ -) than those with ASD. In contrast, those with SZ had significantly lower scores on the active and developmental quality code reflecting synthesized response (DQ+) subscales than those with ASD.

Conclusion: The present findings reveal that individuals with $\mathrm{SZ}$ might have more stress tolerance, stronger perception distortions, and simpler and poorer recognition than those with ASD. We suggest that the Rorschach test might be a useful tool for differentiating between SZ and ASD. Keywords: autism spectrum disorder, perception, psychopathology, Rorschach test, schizophrenia

\section{Introduction}

Schizophrenia (SZ) is a psychotic disorder that begins during adolescence and is typically characterized by positive and negative symptoms. ${ }^{1}$ In addition, $\mathrm{SZ}$ is associated with cognitive impairment that is present before the emergence of the illness hallmark positive symptoms and determines social functional outcomes. ${ }^{2}$ On the other hand, autism spectrum disorder (ASD) is an early-onset neurodevelopmental disorder characterized by impaired social communication skills, social relatedness, and stereotyped behaviors. ${ }^{3}$ ASD displays a wide spectrum of clinical severity from mild social difficulties to severe functional impairments.

Historically, autistic and psychotic disorders have been considered to be related diagnostic entities. In the late 1970s, one innovation separated SZ from ASD into different diagnostic categories using the diagnostic and statistical manual of mental disorders III (DSM-III). ${ }^{4}$ While such approaches based on DSM criteria truly offer many practical advantages to psychiatry, the results of previous studies have suggested the potential mechanisms underlying the neurobiological overlap between SZ and ASD. ${ }^{5-7}$ Consistent with these findings, several lines of epidemiological study have revealed that $30 \%-50 \%$ of patients have comorbid pervasive development disorder preceding the psychotic symptoms of childhood-onset SZ. ${ }^{7}$ Even among adults with 
the onset of schizophrenic psychosis, approximately half of the patients with SZ were reported to have comorbidity with pervasive development disorder. ${ }^{8}$ Hence, there is some difficulty in distinguishing SZ from ASD due to the clinical courses and biological overlaps.

Previous studies have shown similarities and differences between SZ and ASD in clinical features, including perception, affect, and social cognition. Some researchers have demonstrated that there are similar impairments in eye gaze on faces in social scenes, ${ }^{9}$ theory of mind, ${ }^{10,11}$ judgments of facial trustworthiness, and the perception of emotion. ${ }^{12}$ Other researchers have found that patients with ASD showed greater impairments in social orienting ${ }^{9}$ and facial affect recognition ${ }^{13}$ than did patients with SZ. Of course, differential diagnosis might be accomplished by the careful collection of information about the developmental history of each patient. However, detailed information on early development, which is essential for ASD diagnosis, is often hard to obtain because some children with ASD do not receive a correct diagnosis until adulthood due to the mildness of autistic symptoms and the lack of definite intellectual deficit. ${ }^{14-16}$ Therefore, in adult patients, it is often difficult to differentiate between SZ and ASD because of their shared symptoms. ${ }^{17}$

The Rorschach has long been one of the best known and most widely used projective tests. In this test, a person is shown a series of ten inkblots printed on cards, and then they are required to explain what they see. In psychiatry, the Rorschach test is used to analyze a person's subconscious personality characteristics and emotional functioning. Thus, it is generally applied to detect underlying thought disorders, especially among patients with psychosis. Indeed, individuals with SZ have manifested simplistic Rorschach responses along a number of dimensions. ${ }^{18}$ Moreover, previous studies have reported that there are some similarities in thought disturbances occurring in both SZ and ASD. ${ }^{19}$ Given the nature of the Rorschach, understanding the test's tendencies and differences when administered to individuals with SZ and ASD may be useful in ensuring proper diagnosis of these disorders. However, no direct comparison of both diagnoses has been performed to identify such differences and commonalities.

Consequently, we conducted the Rorschach test to compare the psychological profiles in age, sex, and intelligence quotient-matched young adults with SZ and ASD. We also assessed social, occupational, and psychological functioning using the global assessment of functioning (GAF). SZ and ASD clinical symptoms were evaluated using the positive and negative symptom scale (PANSS) and the autism-spectrum quotient Japanese (AQ-J), respectively.

\section{Methods Participants}

We recruited $20 \mathrm{SZ}$ (12 males: mean \pm SD age $24.9 \pm 6.5$ years; eight females: mean \pm SD age $27.8 \pm 3.3$ years) and ASD (14 males: mean \pm SD age $26.2 \pm 4.8$ years; six females: mean \pm SD age $26.2 \pm 5.0$ years) patients from the outpatient clinics at the Department of Psychiatry, Nara Medical University and Kyo Mental Clinic, Nara, Japan. At least two experienced psychiatrists reached consensus concerning DSM-IV-TR, diagnosis of SZ and ASD (autistic disorder, Asperger disorder, or PDD - not otherwise specified) for each subject, based on structured interviews and reviews of patient medical records. The exclusion criteria included neurological disorders, history of traumatic brain injury and electroconvulsive therapy, serious medical conditions, or any psychiatric comorbidity. The patients' intellectual levels were assessed using the Wechsler adult intelligence scale-third edition. Those who demonstrated a score $<70$ on the fullscale IQ were excluded from the present study by a trained psychologist. Sixteen patients with SZ received neuroleptics (daily chlorpromazine [CP] equivalent dose: $459 \pm 583 \mathrm{mg} \mathrm{CP}$ equivalent), while only two patients with ASD received daily doses of 25 or $550 \mathrm{mg}$ CP equivalent of neuroleptics, respectively. Consequently, the SZ group took more $\mathrm{CP}$ equivalent mean dosages for antipsychotics than did the ASD group.

The study and protocols were approved by the Ethics Committee of Nara Medical University, and were followed in accordance with the Declaration of Helsinki. All study participants or their legal guardians provided written informed consent for their participation prior to the start of the study.

\section{The PANSS}

Using the Japanese version of the PANSS, ${ }^{20}$ we evaluated clinical symptoms in the participants with SZ. Specifically, a 7-point Likert scale, in which higher scores indicated greater severity, was used to rate all the items. Subscale scores were calculated using small sets of variables based on the three PANSS domains: positive, negative, and general psychopathological symptoms.

\section{AQ-J}

The AQ-J was developed to quantify autistic traits in research and clinical practice. It is a Japanese version of the original $\mathrm{AQ}$, and has been proven to show acceptable reliability and validity, including the same cutoff point as the original AQ 
for screening normally intelligent young adults with ASD. ${ }^{21-23}$ Briefly, the AQ-J is a self-reporting questionnaire consisting of 50 items rated on a 4-point scale (definitely agree, slightly agree, slightly disagree, and definitely disagree). ${ }^{24}$ It is divided into five subscales (social skill, attention switching, attention to detail, communication, and imagination), consisting of ten items each.

\section{The GAF scale}

We used the GAF scale as a common tool to evaluate SZ and ASD severity. Briefly, the GAF is a numerical scale ranging from 0 to 100 . Higher GAF scores indicate higher global functioning. Thus, it is widely used to evaluate the severity of illness in psychiatry, and it indeed composes Axis V of the DSM-IV-TR. ${ }^{25}$ In this study, two experienced psychiatrists reached consensus on each subject's GAF scores.

\section{Rorschach test comprehensive system}

The Rorschach inkblot test is a projective psychological test consisting of ten inkblots printed on cards. Since the midtwentieth century, it has been commonly used and widely interpreted in clinical practice. To establish standardized rules for administration, scoring, and interpretation, the publication of the Rorschach: a comprehensive system (TRACS) has been developed with its subsequent extensions and revisions. ${ }^{26-29}$ Thus, TRACS is now widely accepted as one of the most reliable and valid approaches to Rorschach interpretation in the psychological community. ${ }^{30}$ In the present study, we used the Exner's comprehensive system (CS) throughout the Rorschach test. ${ }^{31}$ Briefly, two certified psychologists, who were well trained in psychological testing and specifically certified in the CS method, performed the Rorschach test according to the standard administration and scoring procedures of Exner's CS. In addition, the psychologists were blind to the participants' diagnoses before the project implementation, and then independent of the project insofar as they were responsible in any way for patient treatment or assessment.

Using the Japanese version of the CS system, ${ }^{32}$ responses to the inkblots were carefully coded and added to the scoring worksheet under multiple categories. There are seven steps for coding each response because there are seven categories to code; namely: 1) location and developmental quality; 2) determinants; 3) form quality; 4) contents; 5) popular responses; 6) organizational activity ( $Z$ score); and 7) special scores. To interpret and compare the personality aspects, these categories are subsequently clustered into the following seven sides on the structural summary form: 1) capacity for control stress tolerance; 2) interpersonal perception; 3) affective features;
4) self-perception; 5) information processing; 6) cognitive medication; and 7) ideation. To achieve interpretative validity, the present CS study should obtain at least 14 responses from all examinees.

\section{Statistical analyses}

We used a $\chi^{2}$ test to examine the group differences for sex. In addition, demographic characteristics, GAF scores, and Rorschach profiles were statistically compared between patients with SZ and ASD. The variables that fit the normal distribution were compared using Student's $t$-tests while the remaining variables not normally distributed were analyzed using the Mann-Whitney $U$-test. The statistical calculations were performed using PASW Statistics 18.0 J for Windows (SPSS, Tokyo, Japan). Values were expressed as mean \pm standard deviation (SD). Statistical significance was obtained when $P<0.05$.

\section{Results}

\section{Demographic and clinical data}

The demographic characteristics of the study participants are presented in Table 1. There were no significant differences in age ( $t=1.49, d f=38, P=0.93)$, sex $\left(\chi^{2}=0.44, d f=1\right.$, $P=0.74)$, full-scale IQ $(t=-0.87, d f=38, P=0.19)$, or GAF ( $t=1.12, d f=38, P=0.87$ ) between the $\mathrm{SZ}$ and ASD groups. The mean PANSS subscale scores, including positive, negative, and general psychopathology for the SZ patients were $12.3(\mathrm{SD}=4.8), 14.8(\mathrm{SD}=3.2)$, and $19.1(\mathrm{SD}=5.6)$, respectively. The mean AQ-J score was $33.6(\mathrm{SD}=6.0)$ among the ASD patients. Based on the clinical rating scale results, mean PANSS scores appeared to represent symptom

Table I Participant characteristics

\begin{tabular}{|c|c|c|c|c|c|}
\hline & \multicolumn{2}{|c|}{$\begin{array}{l}\text { Patients } \\
\text { with SZ } \\
(n=20)\end{array}$} & \multicolumn{2}{|c|}{$\begin{array}{l}\text { Patients } \\
\text { with ASD } \\
(n=20)\end{array}$} & \multirow[t]{2}{*}{$P$-value } \\
\hline & Mean & SD & Mean & SD & \\
\hline Age (year) & 26.05 & 5.57 & 26.20 & 4.73 & 0.92 \\
\hline Male/female ${ }^{a}$ & $12 / 8$ & & $14 / 6$ & & 0.74 \\
\hline FIQ (WAIS-III) & 86.60 & $1 \mathrm{l} .43$ & 91.90 & $13.8 \mid$ & 0.19 \\
\hline GAF & 64.95 & 6.15 & 64.65 & 4.86 & 0.87 \\
\hline \multicolumn{6}{|l|}{ PANSS subscales } \\
\hline Positive & 12.30 & 4.77 & NA & NA & NA \\
\hline Negative & 14.80 & 3.17 & NA & NA & NA \\
\hline General psychopathology & 19.05 & 5.55 & NA & NA & NA \\
\hline AQ-J & NA & NA & 33.60 & 5.99 & NA \\
\hline
\end{tabular}

Notes: ${ }^{\text {aThe }} \chi^{2}$ test was used for testing group differences. Otherwise, Student's $t$-tests were used.

Abbreviations: AQ-J, autism-spectrum quotient Japanese; ASD, autism spectrum disorder; FIQ (WAIS-III), full-scale IQ score on the Wechsler adult intelligence scalethird edition; GAF, the global assessment of functioning scale; NA, not applicable; PANSS, the positive and negative syndrome scale; SZ, schizophrenia; SD, standard deviation. 
Table 2 Distinct Rorschach variables between patients with SZ and those with ASD

\begin{tabular}{|c|c|c|c|c|c|}
\hline & \multicolumn{2}{|c|}{$\begin{array}{l}\text { Patients with } \\
\text { SZ }(n=20)\end{array}$} & \multicolumn{2}{|c|}{$\begin{array}{l}\text { Patients with } \\
\text { ASD }(n=20)\end{array}$} & \multirow[t]{2}{*}{$P$-value } \\
\hline & Mean & SD & Mean & SD & \\
\hline \multicolumn{6}{|c|}{ Capacity for control stress tolerance } \\
\hline D score & -0.10 & 1.25 & -1.10 & I.7। & 0.04 \\
\hline Adj $D^{a}$ & 0.10 & 0.91 & -0.80 & 1.47 & 0.02 \\
\hline \multicolumn{6}{|c|}{ Interpersonal perception } \\
\hline Active & 3.00 & 2.53 & 5.05 & 3.52 & 0.04 \\
\hline \multicolumn{6}{|c|}{ Information processing } \\
\hline $\mathrm{DQ}++^{\mathrm{a}}$ & 2.30 & 2.02 & 4.80 & 3.33 & 0.01 \\
\hline DQ。 & 19.80 & 8.20 & 15.30 & 5.14 & 0.04 \\
\hline \multicolumn{6}{|c|}{ Cognitive mediation } \\
\hline FQ- & 3.80 & 3.64 & 1.85 & 1.87 & 0.04 \\
\hline
\end{tabular}

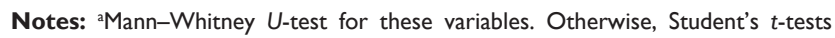
were used.

Abbreviations: Adj D, adjusted D score; ASD, autism spectrum disorder; DQ+, developmental quality code reflecting synthesized response; DQo, developmental quality code reflecting ordinary response; $\mathrm{FQ}-$-, form quality minus; $\mathrm{SZ}$, schizophrenia; SD, standard deviation.

severity approximately corresponding to "mildly ill" among the SZ patients, ${ }^{33}$ and the mean AQ-J score was higher than the cutoff point for the ASD patients. ${ }^{34}$

\section{Distinctive profiles on the Rorschach test between individuals with SZ and ASD}

We found significant differences between the SZ and ASD groups on six Rorschach test variables (Table 2; see also Table S1). The SZ group had significantly higher scores than the ASD group on D score $(t=-1.77, d f=38, P=0.04)$, adjusted D score (Adj D) ( $U=117.00, P=0.02)$, developmental quality code reflecting ordinary response (DQo) $(t=-1.75, d f=38$, $P=0.04)$, and form quality minus (FQ-) $(t=-1.81, d f=38$, $P=0.04)$. In contrast, the $\mathrm{SZ}$ group had significantly lower scores than the ASD group on the active $(t=-1.79, d f=38$, $P=0.04)$ and developmental quality code reflecting synthesized response $(\mathrm{DQ}+)(U=108.50, P=0.01)$ subscales.

\section{Discussion}

Currently, SZ and ASD are classified into different diagnostic categories. However, these disorders often show clinical overlaps and familial coaggregation. In addition, they share genetic and environmental risk factors and cooccur at higher rates, supporting the notion that it is difficult to distinguish between SZ and ASD. In the present study, we sought to determine whether SZ and ASD could show different psychological assessment profiles based on Rorschach test performance. We found statistical differences between the scores of individuals with SZ and ASD on six Rorschach variables. This suggests that the Rorschach test may be helpful in discriminating between SZ and ASD.
Emerging evidence from genetic and neuroimaging studies suggests that both diagnoses share certain common neurobiological processes. ${ }^{5-7,35,36}$ For example, genes with de novo mutations in those with SZ have been shown to partially overlap with genes implicated in ASD. ${ }^{37-39}$ Patients with SZ have been shown to have small brains, while people with ASD often reportedly have larger brains ${ }^{40-43}$ However, previous studies have suggested that the pathophysiology of both diseases is represented as deficits in social brain, ${ }^{44,45}$ which is a specialized neural network system relevant to social cognition, including the prefrontal cortex and superior temporal gyrus. Given the lack of definitive biomarkers in SZ and ASD thus far, a multidimensional assessment based upon biological and psychological aspects might lead to ensuring proper diagnosis of these disorders.

Several researchers have made comparative studies of the clinical manifestations and common characteristics between SZ and ASD. Most studies have shown similarities, ${ }^{46-50}$ and others have revealed subtle differences between the two. ${ }^{51-53}$ Furthermore, SZ and ASD were reported to share certain features of thought pathology, as evaluated by the Rorschach. ${ }^{19}$ In the present study, we found significant differences in six Rorschach response variables on TRACS. According to the literature, ${ }^{32}$ the distinct profile between SZ and ASD could be interpreted as follows. The SZ group had significantly higher scores on D score, Adj D, DQo, and FQ-. As both D score and Adj D are regarded as stress tolerance variables, lower D score and Adj D scores seem to reflect that patients with ASD are more vulnerable to stressors; thus, they are more likely to get confused. The developmental quality (DQ) code refers to the quality of processing involved in forming an answer. Those who have higher DQo scores might prefer the simplicity, and therefore may tend to simplify external stimuli and avoid complicated matters. The FQ is considered an index of perceptual accuracy, indicating that higher FQ scores in patients with SZ might demonstrate stronger perception distortions relative to patients with ASD. In contrast, patients with SZ demonstrate significantly lower scores on the active and DQ+ subscales than do those in the ASD group. Active is one of the determinants and one of the most complex features of scoring the Rorschach. Quite possibly, lower active scores among patients with SZ would reflect lower levels of positive and active attitudes toward interpersonal relationships compared to patients with ASD. DQ+ scores are considered to be part of the process of appropriately analyzing and integrating information. Thus, SZ patients with lower DQ+ scores would have poorer recognition than patients with ASD. Taken together, these data suggest that the psychopathological differences between SZ and ASD 
might be detectable in Rorschach responses, regardless of the current symptomatology.

Our findings are likely to be robust for the following reasons. First, individuals with ASD, except for two patients, were free of psychotropic medication. Although most of the SZ patients were medicated with moderate dosages of antipsychotics, the negative correlation results (all $P>0.08$ ) between the Rorschach variables and the antipsychotic medications (mg CP equivalent) further reduced any potential concerns that our findings might be confounded by antipsychotic drug treatments. Second, GAF scores, as common measures of illness severity, were nearly identical between the patients with SZ and ASD, while the diagnostic validity of these disorders was confirmed using the PANSS or AQ-J. We believe this to be important, given the cases in which psychosocial functioning is similar between patients with SZ and ASD.

The present study has several potential limitations. First, some researchers have questioned the reliability and validity of the Rorschach, ${ }^{54-57}$ although it has been widely used for decades and is an accepted psychometric instrument. In the present study, two certified psychologists who were blind to the participants' diagnoses consistently conducted the Rorschach using TRACS, which is known to be a standardized and structured method of assessment. Nevertheless, we might need to consider differences in cultural or ethnic backgrounds to apply our findings to other countries. ${ }^{58}$ Additional neuropsychological and psychopathological assessments may corroborate our interpretations.

Second, SZ participants received antipsychotics during the experiment. Therefore, we could not rule out the potential effects of the prescribed drugs on the measurements of the Rorschach responses. In previous studies, the presence of thought pathology was reported to be unaltered with illness chronicity and/or effective treatment using antipsychotics, while psychotic symptoms were reduced. ${ }^{59-61}$ As mentioned above, the antipsychotic dosages were not correlated with the Rorschach variables among patients with SZ. This might be relevant when we compare SZ and ASD patients with healthy controls, but unfortunately, this study did not have any healthy comparison subjects. Overall, it would be encouraging to investigate those who are "drug-naïve", or "drug-free". Third, our sample size was relatively small. Future studies with larger samples, including healthy controls, should be conducted to determine whether the different Rorschach responses between patients with SZ and those with ASD are well conserved, and to more thoroughly understand the specific psychopathological aspects.

To the best of our knowledge, this is the first study to compare Rorschach responses between young adults with
SZ and ASD. The present study revealed distinctive profiles concerning the Rorschach responses of individuals with SZ and ASD, suggesting that the Rorschach test might be helpful to aid in the differential diagnosis of SZ and ASD.

\section{Acknowledgments}

We wish to thank the participants for their valuable involvement in this study. We would also like to thank Editage for English language editing.

\section{Disclosure}

The authors report no conflicts of interest in this work.

\section{References}

1. Lieberman JA, Perkins D, Belger A, et al. The early stages of schizophrenia: speculations on pathogenesis, pathophysiology, and therapeutic approaches. Biol Psychiatry. 2001;50(11):884-897.

2. Kahn RS, Keefe RS. Schizophrenia is a cognitive illness: time for a change in focus. JAMA Psychiatry. 2013;70(10):1107-1112.

3. Lai MC, Lombardo MV, Baron-Cohen S. Autism. Lancet. 2014; 383(9920):896-910

4. American Psychological Association (APA). Diagnostic and Statistical Manual of Mental Disorders (3rd ed.), Washington, DC: American Psychological Association (APA); 1980.

5. Cheung C, Yu K, Fung G, et al. Autistic disorders and schizophrenia: related or remote? An anatomical likelihood estimation. PLoS One. 2010;5(8):e12233.

6. King BH, Lord C. Is schizophrenia on the autism spectrum? Brain Res. 2011;1380:34-41.

7. Rapoport J, Chavez A, Greenstein D, Addington A, Gogtay N. Autism spectrum disorders and childhood-onset schizophrenia: clinical and biological contributions to a relation revisited. J Am Acad Child Adolesc Psychiatry. 2009;48(1):10-18.

8. Unenge Hallerback M, Lugnegard T, Gillberg C. Is autism spectrum disorder common in schizophrenia? Psychiatry Res. 2012;198(1): $12-17$

9. Sasson N, Tsuchiya N, Hurley R, et al. Orienting to social stimuli differentiates social cognitive impairment in autism and schizophrenia Neuropsychologia. 2007;45(11):2580-2588.

10. Craig JS, Hatton C, Craig FB, Bentall RP. Persecutory beliefs, attributions and theory of mind: comparison of patients with paranoid delusions, Asperger's syndrome and healthy controls. Schizophr Res. 2004;69(1):29-33.

11. Pilowsky T, Yirmiya N, Arbelle S, Mozes T. Theory of mind abilities of children with schizophrenia, children with autism, and normally developing children. Schizophr Res. 2000;42(2):145-155.

12. Couture SM, Penn DL, Losh M, Adolphs R, Hurley R, Piven J. Comparison of social cognitive functioning in schizophrenia and high functioning autism: more convergence than divergence. Psychol Med.2010; 40(4):569-579.

13. Bolte S, Poustka F. The recognition of facial affect in autistic and schizophrenic subjects and their first-degree relatives. Psychol Med. 2003;33(5):907-915.

14. Bankier B, Lenz G, Gutierrez K, Bach M, Katschnig H. A case of Asperger's syndrome first diagnosed in adulthood. Psychopathology. 1999;32(1):43-46.

15. Ota T, Iida J, Sawada M, et al. Comparison of pervasive developmental disorder and schizophrenia by the Japanese version of the National Adult Reading Test. Int J Psychiatry Clin Pract. 2013;17(1):10-15.

16. Ryan RM. Treatment-resistant chronic mental illness: is it Asperger's syndrome? Hosp Community Psychiatry. 1992;43(8):807-811.

17. Starling J, Dossetor D. Pervasive developmental disorders and psychosis. Curr Psychiatry Rep. 2009;11(3):190-196. 
18. Viglione D. A review of recent research addressing the utility of the Rorschach. Psychol Assessment. 1999;11(3):251-265.

19. Dykens E, Volkmar F, Glick M. Thought disorder in high-functioning autistic adults. J Autism Dev Disord. 1991;21(3):291-301.

20. Kay SR, Fiszbein A, Opler LA. The positive and negative syndrome scale (PANSS) for schizophrenia. Schizophr Bull. 1987;13(2):261-276.

21. Baron-Cohen S, Wheelwright S, Skinner R, Martin J, Clubley E. The autism-spectrum quotient (AQ): evidence from Asperger syndrome/ high-functioning autism, males and females, scientists and mathematicians. J Autism Dev Disord. 2001;31(1):5-17.

22. Kurita H, Koyama T, Osada H. Autism-spectrum quotient-Japanese version and its short forms for screening normally intelligent persons with pervasive developmental disorders. Psychiatry Clin Neurosci. 2005;59(4):490-496.

23. Woodbury-Smith MR, Robinson J, Wheelwright S, Baron-Cohen S. Screening adults for Asperger syndrome using the AQ: a preliminary study of its diagnostic validity in clinical practice. J Autism Dev Disord. 2005;35(3):331-335.

24. Kurita H, Koyama T. Autism-spectrum quotient Japanese version measures mental health problems other than autistic traits. Psychiatry Clin Neurosci. 2006;60(3):373-378.

25. Aas IH. Global Assessment of Functioning (GAF): properties and frontier of current knowledge. Ann Gen Psychiatry. 2010;9:20.

26. Exner J. The Rorschach: A Comprehensive System, Vol. 1 (2nd ed.), Hoboken, NJ: John Wiley \& Sons; 1986.

27. Exner J. The Rorschach: A Comprehensive System, Vol. 2 (2nd ed.), Hoboken, NJ: John Wiley \& Sons; 1991.

28. Exner J. The Rorschach: A Comprehensive System, Vol. 1 (4th ed.), Hoboken, NJ: John Wiley \& Sons; 2003.

29. Exner J, Erdberg P. The Rorschach: A Comprehensive System, Vol. 2, Advanced intepretation (3rd ed.), Hoboken, NJ: John Wiley \& Sons; 2005.

30. Meyer GJ, Hsiao WC, Viglione DJ, Mihura JL, Abraham LM. Rorschach scores in applied clinical practice: a survey of perceived validity by experienced clinicians. J Pers Assess. 2013;95(4):351-365.

31. Takahashi M, Takahashi Y, Nishio H. Implementation method of the Rorschach. Kongou Syuppan. 2006, Tokyo. Japanese.

32. Takahashi M, Takahashi Y, Nishio H. Method of Rorschach interpretation. Kongou Syuppan. 2007, Tokyo. Japanese.

33. Leucht S, Kane JM, Kissling W, et al. What does the PANSS mean? Schizophr Res. 2005;79(2-3):231-238.

34. Kurita H, Koyama T, Osada H. Autism-spectrum quotient-Japanese version and its short forms for screening normally intelligent persons with pervasive developmental disorders. Psychiatry Clin Neurosci. 2005;59(4):490-496.

35. Carroll LS, Owen MJ. Genetic overlap between autism, schizophrenia and bipolar disorder. Genome Med. 2009;1(10):102.

36. Crespi B, Stead P, Elliot M. Evolution in health and medicine Sackler colloquium: comparative genomics of autism and schizophrenia. Proc Natl Acad Sci U S A. 2010;107(Suppl 1):1736-1741.

37. Guilmatre A, Dubourg C, Mosca AL, et al. Recurrent rearrangements in synaptic and neurodevelopmental genes and shared biologic pathways in schizophrenia, autism, and mental retardation. Arch Gen Psychiatry. 2009;66(9):947-956.

38. McCarthy SE, Gillis J, Kramer M, et al. De novo mutations in schizophrenia implicate chromatin remodeling and support a genetic overlap with autism and intellectual disability. Mol Psychiatry. 2014;19(6):652-658.

39. Schizophrenia Working Group of the Psychiatric Genomics Consortium. Biological insights from 108 schizophrenia-associated genetic loci. Nature. 2014;511(7510):421-427.

40. Flashman LA, McAllister TW, Andreasen NC, Saykin AJ. Smaller brain size associated with unawareness of illness in patients with schizophrenia. Am J Psychiatry. 2000;157(7):1167-1169.

41. Stanfield AC, McIntosh AM, Spencer MD, Philip R, Gaur S, Lawrie SM. Towards a neuroanatomy of autism: a systematic review and meta-analysis of structural magnetic resonance imaging studies. Eur Psychiatry. 2008;23(4):289-299.
42. Steen RG, Mull C, McClure R, Hamer RM, Lieberman JA. Brain volume in first-episode schizophrenia: systematic review and meta-analysis of magnetic resonance imaging studies. Br J Psychiatry. 2006;188: $510-518$

43. Vita A, De Peri L, Silenzi C, Dieci M. Brain morphology in first-episode schizophrenia: a meta-analysis of quantitative magnetic resonance imaging studies. Schizophr Res. 2006;82(1):75-88.

44. Burns J. The social brain hypothesis of schizophrenia. World Psychiatry. 2006;5(2):77-81.

45. Pelphrey KA, Shultz S, Hudac CM, Vander Wyk BC. Research review: constraining heterogeneity: the social brain and its development in autism spectrum disorder. J Child Psychol Psychiatry. 2001;52(6):631-644.

46. Barneveld PS, Pieterse J, de Sonneville L, et al. Overlap of autistic and schizotypal traits in adolescents with autism spectrum disorders. Schizophr Res. 2011;126(1-3):231-236.

47. Chung YS, Barch D, Strube M. A meta-analysis of mentalizing impairments in adults with schizophrenia and autism spectrum disorder. Schizophr Bull. 2014;40(3):602-616.

48. Eack SM, Bahorik AL, McKnight SA, et al. Commonalities in social and non-social cognitive impairments in adults with autism spectrum disorder and schizophrenia. Schizophr Res. 2013;148(1-3):24-28.

49. Lugnegard T, Hallerback MU, Gillberg C. Asperger syndrome and schizophrenia: overlap of self-reported autistic traits using the autismspectrum quotient (AQ). Nord J Psychiatry. 2015;69(4):268-274.

50. Mealey A, Abbott G, Byrne LK, McGillivray J. Overlap between autistic and schizotypal personality traits is not accounted for by anxiety and depression. Psychiatry Res. 2014;219(2):380-385.

51. Lugnegard T, Unenge Hallerback M, Hjarthag F, Gillberg C. Social cognition impairments in Asperger syndrome and schizophrenia. Schizophr Res. 2013;14(2-3):277-284.

52. Sasson NJ, Pinkham AE, Weittenhiller LP, Faso DJ, Simpson C. Context effects on facial affect recognition in schizophrenia and autism: behavioral and eye-tracking evidence. Schizophr Bull. 2016;42(3):675-683.

53. Spek AA, Wouters SGM. Autism and schizophrenia in high functioning adults: behavioral differences and overlap. Res Autism Spectr Disord. 2010;198(4):709-717.

54. Grove WM, Barden RC, Garb HN, Lilienfeld SO. Failure of Rorschach-comprehensive-system-based testimony to be admissible under the Daubert-Joiner-Kumho standard. Res Autism Spectr Disord. 2002;8:216-234.

55. Hilsenroth MJ, Cromer TD. Clinician interventions related to alliance during the initial interview and psychological assessment. Psychotherapy (Chic). 2007;44(2):205-218.

56. Mihura JL, Meyer GJ, Dumitrascu N, Bombel G. The validity of individual Rorschach variables: systematic reviews and meta-analyses of the comprehensive system. Psychol Bull. 2013;139(3):548-605.

57. Wood JM, Garb HN, Nezworski MT, Lilienfeld SO, Duke MC. A second look at the validity of widely used Rorschach indices: comment on Mihura, Meyer, Dumitrascu, and Bombel (2013). Psychol Bull. 2015;141(1):236-249.

58. Allen J, Dana RH. Methodological issues in cross-cultural and multicultural Rorschach research. J Pers Assess. 2004;82(2):189-208.

59. Ganellen RJ. Integrating the Rorschach and the MMPI-2 in personality assessment (LEA Series in Personality and Clinical Psychology). Routledge, UK; 1996.

60. Spohn HE, Coyne L, Larson J, Mittleman F, Spray J, Hayes K. Episodic and residual thought pathology in chronic schizophrenics: effect of neuroleptics. Schizophr Bull. 1986;12(3):394-407.

61. Rosenbaum B, Andersen PB, Knudsen PB, Lorentzen P. Rorschach inkblot method data at baseline and after 2 years treatment of consecutively admitted patients with first-episode schizophrenia. Nord J Psychiatry. 2012;66(2):79-85. 


\section{Supplementary material}

Table SI Comparison of Rorschach responses between SZ and ASD on Rorschach test comprehensive system

\begin{tabular}{|c|c|c|c|c|c|}
\hline \multirow[t]{2}{*}{ Seven coding categories } & \multicolumn{2}{|c|}{ Patients with SZ $(n=20)$} & \multicolumn{2}{|c|}{ Patients with ASD $(n=20)$} & \multirow[t]{2}{*}{$P$-value } \\
\hline & Mean & SD & Mean & SD & \\
\hline \multicolumn{6}{|c|}{ Capacity for control stress tolerance } \\
\hline D score & -0.10 & 1.25 & -1.10 & 1.71 & $0.04 *$ \\
\hline Adj $D^{a}$ & 0.10 & 0.91 & -0.80 & 1.47 & $0.02 *$ \\
\hline $\mathrm{R}^{\mathrm{a}}$ & 23.60 & 8.70 & 21.25 & 5.60 & 0.55 \\
\hline L & 2.22 & 2.37 & 2.11 & 3.96 & 0.90 \\
\hline EA & 4.25 & 3.21 & 3.95 & 2.30 & 0.74 \\
\hline es & 4.70 & 3.15 & 7.05 & 4.96 & 0.08 \\
\hline $\mathrm{FM}^{\mathrm{a}}$ & 1.90 & 1.77 & 3.95 & 3.86 & 0.18 \\
\hline $\mathrm{m}^{\mathrm{a}}$ & 0.55 & 0.83 & 1.20 & 1.36 & 0.08 \\
\hline$C^{\prime a}$ & 0.05 & 0.22 & 0 & 0 & 0.32 \\
\hline $\mathrm{T}$ & 0 & 0 & 0 & 0 & NA \\
\hline M & 1.95 & 1.43 & 2.25 & 1.86 & 0.57 \\
\hline V & 0 & 0 & 0 & 0 & NA \\
\hline Y & 0 & 0 & 0 & 0 & NA \\
\hline \multicolumn{6}{|l|}{ Interpersonal perception } \\
\hline Active & 3.00 & 2.53 & 5.05 & 3.52 & $0.04 *$ \\
\hline Passive & 1.50 & 1.36 & 2.35 & 1.93 & 0.12 \\
\hline COP & 0.45 & 2.01 & 0.20 & 0.52 & 0.59 \\
\hline AG & 0.50 & 2.01 & 0.25 & 0.72 & 0.60 \\
\hline GHR & 2.55 & 1.85 & 3.05 & 2.19 & 0.44 \\
\hline PHR & 1.70 & 1.22 & 2.15 & 1.90 & 0.38 \\
\hline Food & 0.65 & $0.8 I$ & 0.70 & 1.13 & 0.87 \\
\hline SumT & 0.25 & 0.44 & 0.05 & 0.22 & 0.08 \\
\hline All $\mathrm{H}$ & 4.15 & 2.16 & 5.10 & 3.19 & 0.28 \\
\hline PureH & 1.65 & 1.34 & 2.10 & 1.62 & 0.35 \\
\hline PER & 0.30 & 0.66 & 0.20 & 0.70 & 0.64 \\
\hline Isol & 0.16 & 0.10 & 0.14 & 0.09 & 0.54 \\
\hline \multicolumn{6}{|l|}{ Affective features } \\
\hline $\mathrm{FC}$ & 1.85 & 1.87 & 1.50 & 1.28 & 0.16 \\
\hline $\mathrm{CF}$ & 0.75 & 1.25 & 1.00 & 1.26 & 0.69 \\
\hline PureC & 0.30 & 1.34 & 0 & 0 & 0.32 \\
\hline SumC' & 1.25 & 1.59 & 1.60 & 1.88 & $0.4 I$ \\
\hline WSumC & 2.25 & 2.70 & 1.70 & 1.29 & 0.06 \\
\hline Afr & 0.44 & 0.17 & 0.47 & 0.20 & 0.58 \\
\hline Blends & 0.75 & 0.97 & 1.25 & 1.11 & 0.14 \\
\hline $\mathrm{CP}$ & 0 & 0 & 0 & 0 & NA \\
\hline \multicolumn{6}{|l|}{ Self-perception } \\
\hline $3 r+(2) / R$ & 0.17 & 0.10 & 0.25 & 0.17 & 0.09 \\
\hline $\mathrm{Fr}^{\mathrm{a}}$ & 0.55 & 2.04 & 0.05 & 0.22 & 0.52 \\
\hline $\mathrm{rF}^{\mathrm{a}}$ & 0.45 & 2.01 & 0 & 0 & 0.32 \\
\hline SumV & 0.15 & 0.37 & 0.10 & 0.31 & 0.35 \\
\hline FD & 0.65 & 2.01 & 0.20 & 0.52 & 0.34 \\
\hline MOR & 0.55 & 0.69 & 0.75 & 1.45 & 0.14 \\
\hline$(\mathrm{H})$ & 0.65 & 1.04 & 0.75 & 0.91 & 0.75 \\
\hline $\mathrm{Hd}$ & 1.45 & 1.01 & 1.45 & 1.43 & 1.00 \\
\hline$H x^{a}$ & 0 & 0 & 0.15 & 0.67 & 0.32 \\
\hline \multicolumn{6}{|l|}{ Information processing } \\
\hline $\mathrm{DQ}++^{\mathrm{a}}$ & 2.30 & 2.02 & 4.80 & 3.33 & $0.01 *$ \\
\hline DQo & 19.80 & 8.20 & 15.30 & 5.14 & $0.04 *$ \\
\hline $\mathrm{DQv} /+^{\mathrm{a}}$ & 0.05 & 0.22 & 0 & 0 & 0.32 \\
\hline DQv & 1.35 & 2.03 & 1.05 & 1.23 & 0.58 \\
\hline$Z f^{a}$ & 14.55 & 7.29 & 14.7 & 4.34 & 0.88 \\
\hline
\end{tabular}


Table SI (Continued)

\begin{tabular}{|c|c|c|c|c|c|}
\hline & \multicolumn{2}{|c|}{ Patients with SZ $(n=20)$} & \multicolumn{2}{|c|}{ Patients with ASD $(n=20)$} & \multirow[t]{2}{*}{$P$-value } \\
\hline & Mean & SD & Mean & SD & \\
\hline$W^{a}$ & 14.40 & 8.20 & 12.75 & 4.48 & 0.59 \\
\hline $\mathrm{D}$ & 6.90 & 5.49 & 7.00 & 5.42 & 0.95 \\
\hline $\mathrm{Dd}^{\mathrm{a}}$ & 3.25 & 4.90 & 1.45 & 1.50 & 0.49 \\
\hline Zd & -1.12 & 5.51 & 0.53 & 3.96 & 0.28 \\
\hline PSV & 0.45 & 0.83 & 0.15 & 0.67 & 0.06 \\
\hline \multicolumn{6}{|c|}{ Cognitive mediation } \\
\hline FQ- & 3.80 & 3.64 & 1.85 & 1.87 & $0.04 *$ \\
\hline $\mathrm{FQ}+$ & 0 & 0 & 0 & 0 & NA \\
\hline FQo & 12.35 & 3.27 & 11.90 & 3.39 & 0.67 \\
\hline $\mathrm{FQu}$ & 7.10 & 4.54 & 7.45 & 4.40 & 0.81 \\
\hline $\mathrm{XA} \%$ & 0.85 & 0.12 & 0.91 & 0.08 & 0.06 \\
\hline WDA\% & 0.87 & 0.09 & 0.91 & 0.09 & 0.22 \\
\hline $\mathrm{X}-\%$ & 0.14 & 0.10 & 0.09 & 0.08 & 0.07 \\
\hline $\mathrm{S}-\%^{\mathrm{a}}$ & 0.80 & I.II & 0.35 & 0.67 & 0.16 \\
\hline$P$ & 3.80 & 1.96 & 3.40 & 1.53 & 0.48 \\
\hline$X+\%$ & 0.54 & 0.20 & 0.57 & 0.12 & 0.51 \\
\hline Xu\% & 0.31 & 0.20 & 0.35 & 0.14 & 0.23 \\
\hline \multicolumn{6}{|l|}{ Ideation } \\
\hline $\mathrm{Ma}$ & 0.95 & 1.23 & 1.45 & 1.36 & 0.21 \\
\hline Mp & 0.95 & 1.01 & 0.95 & 1.23 & 1.00 \\
\hline$M-$ & 0.10 & 0.30 & 0.10 & 0.30 & 1.00 \\
\hline $2 A B+A r t+A y$ & 1.20 & 1.60 & 1.85 & 2.35 & 0.31 \\
\hline MOR & 0.55 & 0.69 & 0.75 & 1.45 & 0.14 \\
\hline Sum6 & 0.50 & 1.47 & 1.15 & 2.01 & 0.25 \\
\hline WSum6 & 1.75 & 5.49 & 3.95 & 7.32 & 0.17 \\
\hline
\end{tabular}

Notes: alann-Whitney $U$-tests for these variables. Otherwise, Student's $t$-tests were used; $* P<0.05$.

Abbreviations: Adj D, adjusted D score; Afr, affective ratio; AG, aggressive movement; All H, all human; ASD, autism spectrum disorder; Blends, blend response; C', pure achromatic color response; CF, color form response; COP, cooperative movement; CP, color projection; D, common detail response; Dd, unusual detail response; DQ+, developmental quality code reflecting synthesized response; DQo, developmental quality code reflecting ordinary response, DQv, Developmental Quality code reflecting vague response; DQv/+, Developmental Quality code reflecting synthesized response; EA, experience actual; es, experienced stimulation; FC, Form-color response; FD, form-based dimensional response; FM, animal movement response; Fr, form-reflection response; FQ+, form quality superior-overelaborated; FQ-, form quality minus; FQo, form quality ordinary; FQu, form quality unusual; GHR, good human representation responses; (H), Whole human (fictional or mythological); Hd, Human detail; Hx, Human experience; Isol, isolation; L, lambda; M, human movement response; $\mathrm{m}$, inanimate movement response; Ma, human movement active; Mp, human movement passive; MOR, morbid content; M-, human movement minus; P, Popular; PER, personalized responses; PHR, poor human representation responses; PSV, perseveration; PureC, pure color response; PureH, whole human response; R, total number of responses to any location in the blot; rF, reflection-form response; S-\%, Space Response minus; SumC', Sum Achromatic Color; SumT, sum texture; SumV, sum vista; Sum6, sum of 6 critical special scores; SZ, schizophrenia; T, pure texture response; V, pure vista response; W, whole response; WDA\%, form appropriate common areas; WSumC: weighted sum color; WSum6, sum weighted values of 6 special scores; $X A \%$, form appropriate extended; $X+\%$, Conventional form use; $X-\%$, distorted form; $X u \%$, usual form use; $Y$, pure shading response; $Z d, Z$ difference; $Z f$, $Z$ frequency; $2 A B+A r t+A y$, intellectualization index; $3 r+(2) / R$, egocentric index.

\section{Publish your work in this journal}

Neuropsychiatric Disease and Treatment is an international, peerreviewed journal of clinical therapeutics and pharmacology focusing on concise rapid reporting of clinical or pre-clinical studies on a range of neuropsychiatric and neurological disorders. This journal is indexed on PubMed Central, the 'PsycINFO' database and CAS, and is the official journal of The International Neuropsychiatric Association (INA). The manuscript management system is completely online and includes a very quick and fair peer-review system, which is all easy to use. Visit http://www.dovepress.com/testimonials.php to read real quotes from published authors. 